Draft VERSion April 21, 2022

Preprint typeset using $\mathrm{IAT}_{\mathrm{E}} \mathrm{X}$ style emulateapj v. 5/2/11

\title{
SPECTRAL ENERGY DISTRIBUTIONS OF ACCRETING PROTOPLANETS
}

\author{
J.A. EISNER ${ }^{1}$ \\ Steward Observatory, The University of Arizona, 933 N. Cherry Ave, Tucson, AZ 85721 \\ Draft version April 21, 2022
}

\begin{abstract}
Planets are often invoked as the cause of inferred gaps or inner clearings in transition disks. These putative planets would interact with the remnant circumstellar disk, accreting gas and generating substantial luminosity. Here I explore the expected appearance of accreting protoplanets at a range of evolutionary states. I compare synthetic spectral energy distributions with the handful of claimed detections of substellar-mass companions in transition disks. While observed fluxes of candidate companions are generally compatible with accreting protoplanets, challenges remain in reconciling the extended structure inferred in observed objects with the compact emission expected from protoplanets or circumplanetary disks. I argue that a large fraction of transition disks should harbor bright protoplanets, and that more may be detected as larger telescopes open up additional parameter space. Subject headings: accretion, accretion disks-protoplanetary disks-planets and satellites: formation - planet-disk interactions
\end{abstract}

\section{INTRODUCTION}

Transition disks exhibit spectral energy distributions (SEDs) similar to classical $\mathrm{T}$ Tauri or Herbig Ae/Be stars, but with a deficit of emission at near-IR wavelengths (e.g., Strom et al. 1989, Calvet et al. 2002). This lack of near-IR excess emission suggests inner clearings. Direct imaging has now confirmed the existence of such inner holes, and indicated sharp edges between the optically thick outer disks and the cleared inner regions (e.g., Hughes et al. 2007; Andrews et al. 2011).

Dynamical interactions of a massive planet and disk can cause sharp edges like those seen in transition disks (e.g., Bryden et al. 1999). Planets may also explain the lower-by up to an order of magnitude - accretion rates inferred for transition disks relative to classical T Tauri stars (Najita et al. 2007; Flaherty \& Muzerolle 2008; Kim et al. 2013). Since transition disk masses are similar to those of $\mathrm{T}$ Tauri stars, lower accretion rates onto the star suggest some of the accretion flow is diverted in the inner regions (Najita et al. 2007). Numerical models of forming planets corroborate the idea that protoplanets can take up a large fraction of the circumstellar accretion flow (e.g., Varnière et al. 2006). Protoplanets may open gaps in less evolved disks as well, as illustrated by the gapped-disk structure seen in HL Tau with ALMA; multiple, low-mass protoplanets can potentially explain such observations (e.g., Dong et al. 2014).

Accreting protoplanets may generate substantial luminosity (e.g., Papaloizou \& Nelson 2005; Zhu 2015):

$$
L_{\mathrm{acc}, \mathrm{p}} \sim \frac{G M_{\mathrm{p}} \dot{M}_{\mathrm{p}}}{R_{\mathrm{p}}} .
$$

Here the $p$ subscripts indicate quantities related to the protoplanet. For planets of Jupiter mass and radius, and accretion rates of $\dot{M}_{\mathrm{p}} \sim 10^{-9} \mathrm{M}_{\odot} \mathrm{yr}^{-1}(\sim 10 \%$ of typical accretion rates onto $\mathrm{T}$ Tauri stars), $L_{\text {acc, }}>10^{-4} \mathrm{~L}_{\odot}$.

\footnotetext{
jeisner@email.arizona.edu

${ }^{1}$ Visiting Fellow, JILA, University of Colorado and NIST, Boulder, CO 80309
}

However protoplanets may be much larger than $R_{\mathrm{J}}$ at early times, in which case a given $\dot{M}$ would produce less luminosity.

Here I estimate the appearance of accreting protoplanets during different evolutionary stages, assuming formation via core accretion. Zhu 2015) recently computed synthetic SEDs for circumplanetary accretion disks. I argue that such models are only appropriate after protoplanet atmospheres have undergone hydrodynamic collapse, and I constrain the lifetime over which this evolutionary phase may be observed. I discuss the potential of current observational surveys to detect accreting protoplanets at a range of evolutionary stages.

\section{ACCRETION ONTO PROTOPLANETS}

Planet accretion begins with the assembly of a solid core. If cores attain sufficient mass $\left(\gtrsim 10 \mathrm{M}_{\oplus}\right.$; e.g., Pollack et al. 1996), the gravity of the protoplanet is sufticient to overcome the tidal gravity of the central star in a substantial "feeding zone", roughly the size of the Hill sphere:

$$
R_{\mathrm{H}}=a\left(\frac{M_{\mathrm{p}}}{3 M_{*}}\right)^{1 / 3} .
$$

As planets accrete mass, the Hill radius grows, and for a Jupiter-mass planet at $5 \mathrm{AU}$ around a solar-type star, $R_{\mathrm{H}} \approx 750 \mathrm{R}_{\mathrm{J}}(\approx 0.35 \mathrm{AU})$.

The scale height of circumstellar disks (at a few AU) is similar to or larger than the size of the Hill sphere for forming gas giants (see Section 3), leading to spherical accretion onto protoplanets. At early times protoplanet envelopes are in hydrostatic equilibrium, with energy liberated by infalling planetesimals providing pressure support against gravity. The high opacities in protoplanet atmospheres result in hydrostatic envelopes filling a large fraction of the Hill sphere (e.g., Pollack et al. 1996). Because $R_{\mathrm{H}}>100 \mathrm{R}_{\mathrm{J}}$, gas does not accrete deep into the potential well of the protoplanet. Although the mass accretion rate of planetesimals is about an order of magnitude lower than the gas accretion rate during this hydrostatic stage, the luminosity is dominated by planetes- 

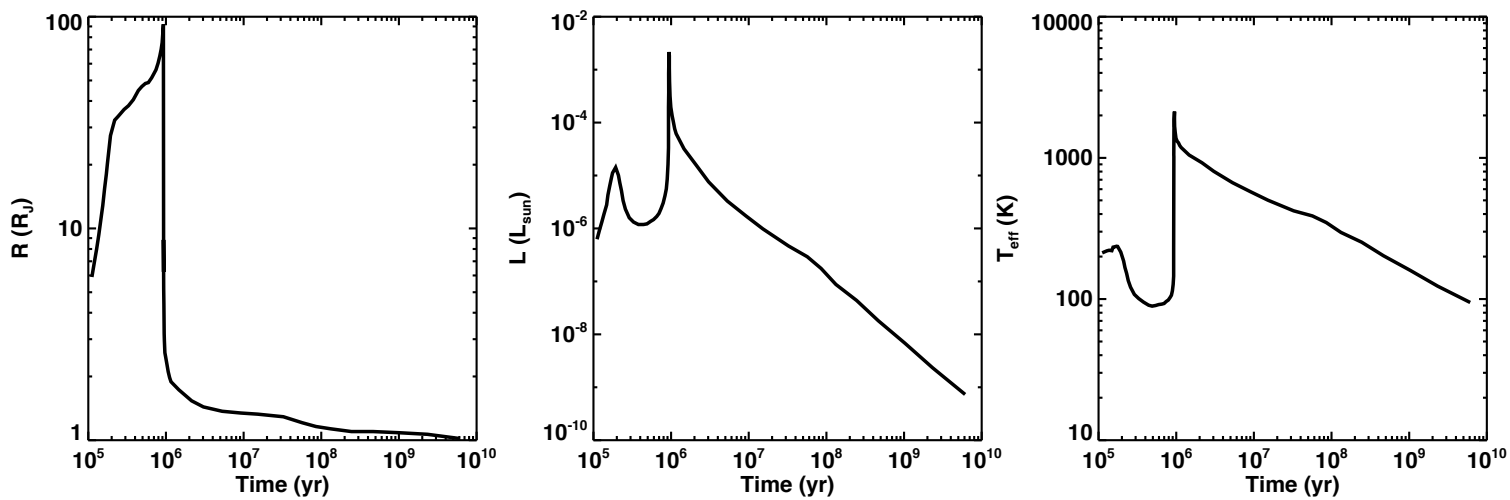

FIG. 1.- Radii, luminosities, and effective temperatures for a giant planet undergoing gas accretion to eventually reach $\mathrm{M}_{\mathrm{J}}$. $R$ and $L$ are taken from Mordasini et al. (2012), and I used these to calculate $T_{\text {eff }}$.

imals, since these penetrate deeper into the protoplanet envelope (Pollack et al. 1996).

Protoplanets in hydrostatic equilibrium accrete gas slowly, since they must cool and contract in order to allow more matter into the Hill sphere. However once the mass of the gaseous envelope reaches the "crossover mass" $\left(M_{\text {env }} \approx M_{\text {core }} \approx 10-20 \mathrm{M}_{\oplus}\right)$, hydrostatic equilibrium breaks down and the atmosphere collapses (e.g., Pollack et al. 1996). Numerical simulations suggest that after hydrodynamic collapse, a circumplanetary disk is formed within a fraction of the Hill radius (e.g., Ayliffe \& Bate 2012). At this stage, the circumplanetary disk can bring matter down close to $R_{\mathrm{J}}$, deep in the potential well of the protoplanet, and high accretion luminosities are possible (e.g., Equation 1; Papaloizou \& Nelson 2005).

Pollack et al. (1996) predict that accreting protoplanets will have hydrostatically supported envelopes for $\sim 7$ Myr. This timescale is uncomfortably long compared to observed lifetimes of gaseous circumstellar disks (e.g., Fedele et al. 2010). Planetary migration can accelerate the accretion process, since the feeding zone continually takes in new matter (Alibert et al. 2005). Revised opacities for forming giant planet atmospheres also result in substantially accelerated accretion, because energy can be radiated away more efficiently (e.g., Movshovitz et al. 2010). In these cases, the envelope reaches the crossover mass - after which hydrodynamic collapse leads to runaway gas accretion - in approximately a tenth the time required in the Pollack et al. (1996) models.

After circumplanetary disk formation, the accretion rate onto the protoplanet is limited by how fast the circumstellar disk can supply material. Circumstellar accretion rates decline on $\sim 10^{6} \mathrm{yr}$ timescales (e.g., Fedele et al. 2010), suggesting that high accretion rates onto protoplanets - which do not undergo hydrodynamic collapse until $\sim 10^{6} \mathrm{yr}$ - are not likely to continue long. Furthermore, any objects that continued to accrete at such rates for $\gtrsim 1 \mathrm{Myr}$ would exceed the planetary mass range. The observed accretion rates onto the central stars in transition disk systems are $\lesssim 10^{-9} \mathrm{M}_{\odot} \mathrm{yr}^{-1}$, which may indicate a large fraction of the accretion flow is intercepted by protoplanets with $\dot{M}_{\mathrm{p}}$ near the runaway accretion rate (e.g., Najita et al. 2007, Varnière et al. 2006). However, the stellar accretion rates can also be naturally explained with MRI-driven viscosity (Chiang \& Murray-Clay 2007). It is thus unclear how much proto- planetary accretion can be currently sustained in known transition disks.

\section{GAP OPENING}

Protoplanets whose Hill radii are comparable to the scale height of the circumstellar disk can open gaps via dynamical processes (e.g., Lin \& Papaloizou 1993). Such gaps are presumably linked with the appearance of transition disks, and also render protoplanets easier to observe.

To determine when protoplanets might open gaps, I first estimate the circumstellar disk scale height. For a flared disk around a typical $\mathrm{T}$ Tauri star, $H / R \sim 0.05$ 0.1 within $\sim 10 \mathrm{AU}$ (e.g., Chiang \& Goldreich 1997). However, disk shadowing could lead to substantially smaller scale heights (e.g., Dullemond et al. 2001). A lower bound can be estimated by assuming that the disk is heated only by accretion. For a constant $\dot{M}=10^{-8}$ $\mathrm{M}_{\odot} \mathrm{yr}^{-1}$, the disk temperature is $<100 \mathrm{~K}$ beyond 1 $\mathrm{AU}$, the sound speed is $<0.6 \mathrm{~km} \mathrm{~s}^{-1}$ (assuming the gas is mostly $\left.\mathrm{H}_{2}\right)$ and $H / R \sim c_{\mathrm{s}} / v_{\mathrm{K}} \lesssim 0.025$ within $\sim 10$ AU.

Before hydrodynamic collapse, the total mass of a protoplanet is $\lesssim 20 \mathrm{M}_{\oplus}$ (e.g., Pollack et al. 1996; Mordasini et al. 2012), and thus the Hill radius is $\lesssim 0.03 a$. This is likely smaller than the circumstellar disk scale height, and so the protoplanet is still embedded in the disk at this stage. Indeed, the protoplanet is "attached" to the disk, with continuous pressure and temperature across the disk/protoplanet boundary (e.g., Mordasini et al. 2012). Accretion luminosity generated by hydrostatic protoplanets would thus be attenuated by surface layers of the circumstellar disk.

When hydrodynamic collapse begins, the protoplanet envelope detaches from the circumstellar disk, and forms a circumplanetary disk (e.g., Ayliffe \& Bate 2012, Papaloizou \& Nelson 2005). Near the beginning of the runaway accretion phase, the protoplanet mass is $\sim 90 \mathrm{M}_{\oplus}$ (Mordasini et al. 2012), and $R_{\mathrm{H}} \sim 0.05 a$, probably sufficient for gap opening.

While protoplanets may open gaps in disks, making cleared regions as large as those observed in transition disks is difficult from a theoretical perspective. Multiple planets have been invoked to explain large clearings (Zhu et al. 2011; Dodson-Robinson \& Salyk 2011), but such models can not reproduce both the depleted regions and the observed accretion rates onto the central stars. 
Dust filtration through local pressure maxima created by even small disk gaps may also help to deplete the dust population in large clearings (e.g., Rice et al. 2006; Zhu et al. 2012), although this mechanism fails to clear out small dust grains. Radiation pressure, either from the central star (e.g., Eisner et al. 2006) or from the accreting protoplanet itself (Owen 2014) may help to clear out the small dust.

Since several different physical mechanisms may be responsible for creating or maintaining the cleared regions in transition disks, one should be cautious about the exact criteria used to determine when protoplanets open gaps. For example, any non-monotonic pressure profile associated with a protoplanet may provide a seed for other physical mechanisms to clear a gap. It is thus difficult to be certain that hydrostatic protoplanetsespecially as they grow in mass and luminosity at later times - will be completely enshrouded by circumstellar disk matter. Furthermore, if lower-mass protoplanets do not open deep gaps like those seen in transition disks, they may open smaller gaps. Evidence for this is seen in the HL Tau disk, where the gapped disk structure observed with ALMA can be explained with multiple, $\sim 60$ $\mathrm{M}_{\oplus}$ protoplanets (Dong et al. 2014).

\section{PROTOPLANET SEDS OVER TIME}

To predict the appearance of accreting protoplanets, I use calculations of the envelope structure of a forming giant planet (e.g., Pollack et al. 1996, Mordasini et al. 2012). I consider the first $10 \mathrm{Myr}$ or so, because known transition disks have typical ages of a few Myr, with the oldest at $\sim 10$ Myr. I adopt the models of Mordasini et al. (2012), which predict that runaway accretion occurs after $\sim 0.95 \mathrm{Myr}$. While longer timescales for the onset of runaway accretion are possible (e.g., Pollack et al. 1996), the exact timescale does not affect the results for pre- or post-runaway accretion stages.

Using planetary radii and accretion luminosities calculated as a function of time (Mordasini et al. 2012), I determine effective temperatures (Figure 1). Radii and effective temperatures are then used to generate blackbody SEDs as a function of time. All models are at an assumed distance of $100 \mathrm{pc}$.

Before hydrodynamic collapse, $R_{\mathrm{p}} \approx R_{\mathrm{H}} / 3 \approx 100 \mathrm{R}_{\mathrm{J}}$ (e.g., Mordasini et al. 2012, Lissauer et al. 2009). This large radius, combined with a modest accretion luminosity of $\sim 10^{-6} \mathrm{~L}_{\odot}$, produces cool spectra, with peak wavelengths $>10 \mu \mathrm{m}$ (Figure 2). Since these protoplanets are likely still attached to circumstellar disks, their spectra will probably suffer additional extinction from matter in the circumstellar disk surface layers.

After hydrodynamic collapse, when protoplanets detach from circumstellar disks and probably open gaps, $R_{\mathrm{p}}$ approaches $R_{\mathrm{J}}$, the accretion rate increases, and the luminosity climbs to $\sim 10^{-3} \mathrm{~L}_{\odot}$. This leads to a bright protoplanet with $T_{\text {eff }}>10^{3} \mathrm{~K}$ (Figure 2). The models of Mordasini et al. (2012) assume that runaway accretion occurs for $\sim 10^{5}$ years, until the planet reaches a Jupitermass, after which the circumstellar disk disappears and accretion ceases. After this, the luminosity declines from $\sim 10^{-3} \mathrm{~L}_{\odot}$ to $\sim 10^{-6} \mathrm{~L}_{\odot}$, and $T_{\text {eff }}$ declines from $\sim 10^{3}$ to $\sim 600 \mathrm{~K}$, within $\sim 10 \mathrm{Myr}$, similar to "hot-start" planetary atmosphere models (e.g., Spiegel \& Burrows 2012 .

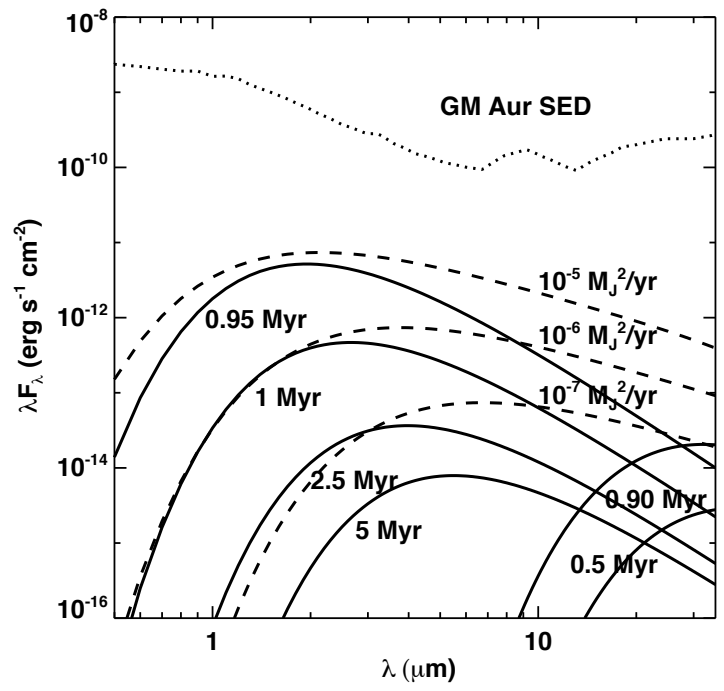

FIG. 2.- SEDs calculated for the accreting protoplanet shown in Figure 1 (solid curves). Several illustrative timesteps are shown, with particular focus on the hydrodynamic collapse of the atmosphere at $\sim 0.95 \mathrm{Myr}$. These models assume that all luminosity is radiated from the protoplanetary surface. Models where the luminosity is generated in circumplanetary disks are shown with dashed curves, labeled by the product of planet mass and accretion rate. I also include the observed SED of the transition disk GM Aur (adjusted to a distance of $100 \mathrm{pc}$; adapted from Zhu 2015), to demonstrate the contrast between protoplanet and central star+circumstellar disk.

These calculations assume that all luminosity is radiated from the protoplanetary radius. However hydrodynamic collapse likely forms a circumplanetary disk (Papaloizou \& Nelson 2005, Ayliffe \& Bate 2012), which would produce accretion luminosity from somewhat larger radii. Zhu (2015) recently computed SEDs for circumplanetary disks with accretion rates similar to those expected for runaway accretion. I reproduce similar models here using a simplified treatment. Considering a disk extending from $2 R_{\mathrm{J}}$ to $R_{\mathrm{H}} / 3$, I compute blackbody SEDs for a series of annuli and sum them. SEDs for disks around a Jupiter-mass planet with $\dot{M}_{\mathrm{p}}$ between $10^{-5}$ and $10^{-7} \mathrm{M}_{\mathrm{J} \mathrm{yr}} \mathrm{yr}^{-1}$ are shown in Figure 2 These curves are nearly identical to those in Zhu $(20 \overline{15})$.

A circumplanetary accretion disk with $M M_{\mathrm{p}}=10^{-5}$ $\mathrm{M}_{\mathrm{J}}^{2} \mathrm{yr}^{-1}$ produces a similar luminosity to the calculated SED of a protoplanet undergoing runaway accretion. The main difference between the two models is that the disk model reprocesses light at longer wavelengths, leading to a slower falloff in flux (see also Zhu 2015). As protoplanets cool, their luminosities decline (see curves for $\geq 1 \mathrm{Myr}$ in Figure 2p. If circumstellar and circumplanetary accretion continues at these later times, but perhaps declines from its peak rate, then the SEDs may manifest additional emission, particularly at longer wavelengths (see the $10^{-6}$ and $10^{-7} \mathrm{M}_{\mathrm{J}}^{2} \mathrm{yr}^{-1}$ curves in Figure 2).

Whether accretion luminosity is generated at protoplanetary surfaces or in accretion disks, the infrared emission is compact. For $\lambda<5 \mu \mathrm{m}$, any observed emission would be more compact than $\sim 0.01 \mathrm{AU}$. At wavelengths up to $30 \mu \mathrm{m}$, emission may be distributed on scales several times larger.

\section{COMPARISON WITH OBSERVATIONS}


Contrast ratios between modeled protoplanets (Figure 2 and a typical transition disk (GM Aur) are listed in Table 1. Contrasts could be lower for less evolved gapped-disks like HL Tau, which exhibit stronger nearIR excess emission than transition disks. However this additional near-IR emission would be less important for protoplanets at large stellocentric radii.

Extreme adaptive optics on large telescopes can reach contrasts of $\sim 10^{-6}$ at $1^{\prime \prime}$ separations (e.g., Skemer et al. 2014), but such scales are larger than typical transition disk holes. Within 0.'1 (10 AU at 100 pc) achieved contrasts are closer to $10^{-3}$ (e.g., Kraus \& Ireland 2012 Biller et al. 2014. Sallum et al. 2015). Thus, $10^{-3}$ is a reasonable benchmark against which to compare the computed contrast ratios of protoplanet models.

Detecting hydrostatic protoplanets is extremely difficult at the expected contrasts. If emission from a protoplanet is reprocessed by the atmosphere of the circumstellar disk (Section 3), the contrasts at infrared wavelengths may even be more challenging than implied by Table 1. The best hope for detecting hydrostatic protoplanets is observing at long IR wavelengths ${ }^{2}$, and targeting transition disks with particularly large clearings (where observations can achieve higher contrasts).

Protoplanets in, or shortly after, the runaway accretion phase are more easily detectable. Modeled protoplanets with $\dot{M}$ near the runaway accretion rate of $10^{-5} \mathrm{M}_{\mathrm{J}} \mathrm{yr}^{-1}$ all have contrasts of $\sim 10^{-2}$ with the transition disk SED at some wavelength between 2 and $5 \mu \mathrm{m}$ (Table 1). After runaway accretion as the circumstellar and circumplanetary accretion rates decline, protoplanets may fall below detection thresholds.

The most promising candidate is $\mathrm{LkCa} 15$, where localized structure at both $K$ and $L$ bands is detected and inferred to orbit around the central star over time (Kraus \& Ireland 2012). The contrasts with the central star (plus its transition disk) are $\sim 2 \times 10^{-3}$ at $K$ and $\sim 7 \times 10^{-3}$ ar $L$. Similar observations of T Cha suggest the presence of some structure at $K$ and $L$ bands within the cleared region of the disk, with contrasts of $\sim 5 \times 10^{-3}$ at $K$ and $\sim 8 \times 10^{-3}$ at $L$ Huélamo et al. 2011, Sallum et al. 2015). These contrasts, for both sources, are similar to expectations for a protoplanet that has recently undergone runaway accretion (Table 1).

However images of the emission within the cleared regions of both the LkCa 15 and $\mathrm{T}$ Cha transitions disks show complex structure that does not appear to trace compact sources. The maximum outer radius of circumplanetary disks is $\sim R_{\mathrm{H}} / 3 \approx 0.5 \mathrm{AU}$ for even a 10 Jupiter-mass planet within $10 \mathrm{AU}$ of a sun-like star. Moreover, infrared emission is generated in the inner regions of circumplanetary disks $(\lesssim 0.01 \mathrm{AU})$. In contrast, extended structure is seen on $\sim 5$ AU scales in $\mathrm{LkCa}$ 15 (Kraus \& Ireland 2012), and even larger scales in the $\mathrm{T}$ Cha disk (Sallum \& Eisner in prep). While multiple protoplanets might explain the observed structures (and may also help explain the origin of the large cleared inner region in the LkCa 15 disk), disk-planet interactions may also lead to extended, asymmetric structure in transition disks (e.g., Lyra et al. 2009; Fouchet et al. 2010). Future

2 Contrasts are higher in the (sub-)mm due to the luminous long-wavelength SEDs of transition disks. observations at higher resolution are needed to elucidate the nature of the emission.

Coronographic imaging observations suggest a companion in the HD 169142 transition disk, with a detection at only $L$ band, and non-detections at $J, H$, and $K$ (Reggiani et al. 2014; Biller et al. 2014). This may be a case where the protoplanet's contrast with the transition disk SED only exceeds the detection threshold at certain wavelengths. For example, the $\dot{M} M_{\mathrm{p}}=10^{-6} \mathrm{M}_{\mathrm{J}}^{2}$ $\mathrm{yr}^{-1}$ model has contrast of $\sim 10^{-3}$ at $K$ band, but $4-6$ $\times 10^{-3}$ at $L$ and $M$ bands. The inferred contrast of the companion is $\sim 3 \times 10^{-3}$ at $L$ band, matched reasonably well with the model prediction (see also Zhu 2015).

A spatially extended ( $\gtrsim 12$ AU diameter) companion has been claimed in the HD 100546 disk at a stellocentric radius of $\sim 70 \mathrm{AU}$ (Quanz et al. 2013; Currie et al. 2014). Quanz et al. (2014) confirm this companion at both $L$ and $M$ bands, and argue for a compact source surrounded by extended emission. The compact object has a contrast of $\sim 2 \times 10^{-4}$ at both $L$ and $M$ bands (Quanz et al. 2014), consistent with a cooling protoplanet at $\sim 2.5 \mathrm{Myr}$, or a circumplanetary disk with $\dot{M} M_{\mathrm{p}} \sim 10^{-7} \mathrm{M}_{\mathrm{J}}^{2} \mathrm{yr}^{-1}$. However the putative protoplanet appears to be still embedded in the circumstellar disk, suggesting a younger evolutionary state.

Finally, mid-IR imaging has suggested the presence of self-luminous companions in two other transition disks, SR 21 (Eisner et al. 2009) and TW Hya Arnold et al. 2012). The posited companion in the SR 21 disk is too red and luminous compared to the models presented here, suggesting it is unlikely to be an accreting protoplanet. The companion suggested around TW Hya has a luminosity similar to the $\dot{M} M_{\mathrm{p}}=10^{-6} \mathrm{M}_{\mathrm{J}}^{2} \mathrm{yr}^{-1}$ model, but a redder spectral shape. If this companion is real, the accretion would have to fall in only to $\sim 10 \mathrm{R}_{\mathrm{J}}$ to explain the color. Such a large inner disk radius could result from a highly magnetized protoplanet, whose magnetosphere would truncate the disk. Magnetospheric accretion in this putative protoplanet could also lead to high $\mathrm{H} \alpha$ luminosity (Zhu 2015), potentially detectable in follow-up observations at visible wavelengths (e.g., Close et al. 2014).

The models presented here can be used to estimate the probability that accreting proptoplanets would be detected in any transition disk. For current detection thresholds, Jupiter-mass protoplanets are detectable during the runaway accretion phase and for $\sim 0.5$ Myr after, as they cool (Table 1). Higher mass planets stay in the runaway accretion stage longer and start cooling from a higher temperature: a $10 \mathrm{M}_{\mathrm{J}}$ planet might remain detectable for $\sim 1 \mathrm{Myr}$. Taking the circumstellar disk lifetime to be 3 Myr (e.g., Fedele et al. 2010), one expects $\sim 15-30 \%$ of disks to harbor bright protoplanets. This is similar to the percentage of transition disks around young stars 10-30\% (e.g., Muzerolle et al. 2010, Andrews et al. 2011). Thus, it seems likely that a large fraction of these transition disks would host a protoplanet with a high accretion luminosity.

While many transition disks have no detected planets despite observations with the required contrast (e.g., Kraus et al. 2011; Evans et al. 2012), observations probed a limited semimajor axis range. Most candidate protoplanets have semimajor axes $\gtrsim 10$ AU, located near 
TABLE 1

Protoplanet/Transition Disk Contrast Ratios

\begin{tabular}{lcccccc}
\hline \hline \multicolumn{1}{c}{ Model } & $2 \mu \mathrm{m}$ & $4 \mu \mathrm{m}$ & $5 \mu \mathrm{m}$ & $10 \mu \mathrm{m}$ & $20 \mu \mathrm{m}$ & $30 \mu \mathrm{m}$ \\
\hline $\mathbf{0 . 5} \mathbf{M y r}$ & $<10^{-29}$ & $<10^{-17}$ & $<10^{-13}$ & $<10^{-8}$ & $<10^{-6}$ & $<10^{-5}$ \\
$\mathbf{0 . 9} \mathbf{M y r}$ & $<10^{-22}$ & $<10^{-12}$ & $<10^{-10}$ & $<10^{-6}$ & $<10^{-4}$ & $<10^{-4}$ \\
$\mathbf{0 . 9 5} \mathbf{M y r}$ & $10^{-2}$ & $10^{-2}$ & $10^{-2}$ & $10^{-3}$ & $10^{-4}$ & $10^{-4}$ \\
$\dot{M} M_{\mathrm{p}}=10^{-5}$ & $10^{-2}$ & $10^{-2}$ & $10^{-2}$ & $10^{-2}$ & $10^{-3}$ & $10^{-3}$ \\
$\mathbf{1} \mathbf{M} \mathbf{y r}$ & $10^{-3}$ & $10^{-3}$ & $10^{-3}$ & $10^{-4}$ & $10^{-4}$ & $10^{-5}$ \\
$\dot{M} M_{\mathrm{p}}=10^{-6}$ & $10^{-3}$ & $10^{-3}$ & $10^{-2}$ & $10^{-3}$ & $10^{-3}$ & $10^{-4}$ \\
$\mathbf{2 . 5} \mathbf{M y r}$ & $10^{-5}$ & $10^{-4}$ & $10^{-4}$ & $10^{-4}$ & $10^{-5}$ & $10^{-6}$ \\
$\dot{M} M_{\mathrm{p}}=10^{-7}$ & $10^{-4}$ & $10^{-4}$ & $10^{-3}$ & $10^{-4}$ & $10^{-4}$ & $10^{-4}$ \\
$\mathbf{5} \mathbf{M y r}$ & $10^{-6}$ & $10^{-5}$ & $10^{-4}$ & $10^{-5}$ & $10^{-5}$ & $10^{-6}$ \\
& & & & & &
\end{tabular}

Note. - Models in bold assume hydrodynamic collapse and runaway accretion around $0.95 \mathrm{Myr}$, and then $\dot{M}=0$ for $>1 \mathrm{Myr}$. For comparison, I also include circumplanetary accretion disk models with $\dot{M}$ declining from the peak runaway rate. Since protoplanets may still be embedded in circumstellar disks at $<0.95$ Myr, the contrasts are listed as upper limits for such objects.

$\lambda / D$ for the telescopes used in their detections. As larger telescopes become available, smaller separations can be probed, and more accreting protoplanets may be found.

\section{SUMMARY}

Protoplanets that are still hydrostatically supported and attached to their circumstellar disks are faint, red, and may be extincted by circumstellar disk surface layers. Detecting such objects is extremely difficult, although observations at long-IR wavelengths are the best hope. In contrast, protoplanets whose atmospheres have undergone hydrodynamic collapse may be detectable during their runaway accretion phase. Whether accretion lumi- nosity is assumed to be generated at the surface of the protoplanet or in a circumplanetary disk, such objects appear relatively bright compared to the central stars and circumstellar disks. Their infrared emission should be compact, at odds with most claimed observations of protoplanetary companions. While the lifetime of the runaway accretion phase is short compared to circumstellar disk lifetimes, I argue that protoplanets should be detectable in a large fraction of transition disks, and that as larger telescopes probe smaller separations additional protoplanets will be found.

This work was supported by NSF AAG grant 1211329, and benefitted from discussions with Phil Armitage.

\section{REFERENCES}

Alibert, Y., Mordasini, C., Benz, W., \& Winisdoerffer, C. 2005, A\&A, 434, 343

Andrews, S. M., Wilner, D. J., Espaillat, C., et al. 2011, ApJ, 732,42

Arnold, T. J., Eisner, J. A., Monnier, J. D., \& Tuthill, P. 2012, ApJ, 750, 119

Ayliffe, B. A. \& Bate, M. R. 2012, MNRAS, 427, 2597

Biller, B. A., Males, J., Rodigas, et al. 2014, ApJ, 792, L22

Bryden, G., Chen, X., Lin, D. N. C., Nelson, R. P., \& Papaloizou, J. C. B. 1999, ApJ, 514, 344

Calvet, N., D’Alessio, P., Hartmann, L., Wilner, D., Walsh, A., \& Sitko, M. 2002, ApJ, 568, 1008

Chiang, E. \& Murray-Clay, R. 2007, Nature Physics, 3, 604

Chiang, E. I. \& Goldreich, P. 1997, ApJ, 490, 368

Close, L. M., Follette, K. B., Males, et al. 2014, ApJ, 781, L30

Currie, T., Muto, T., Kudo, T., et al. 2014, ApJ, 796, L30

Dodson-Robinson, S. E. \& Salyk, C. 2011, ApJ, 738, 131

Dong, R., Zhu, Z., \& Whitney, B. 2014, arXiv:1411.6063

Dullemond, C. P., Dominik, C., \& Natta, A. 2001, ApJ, 560, 957

Eisner, J. A., Chiang, E. I., \& Hillenbrand, L. A. 2006, ApJ, 637, L133

Eisner, J. A., Monnier, J. D., Tuthill, P., \& Lacour, S. 2009, ApJ, 698, L169

Evans, T. M., Ireland, M., Kraus, A., et al. 2012, ApJ, 744, 120

Fedele, D., van den Ancker, M. E., Henning, T., Jayawardhana, R., \& Oliveira, J. M. 2010, A\&A, 510, A72

Flaherty, K. M. \& Muzerolle, J. 2008, AJ, 135, 966

Fouchet, L., Gonzalez, J.-F., \& Maddison, S. 2010, A\&A, 518, A16

Huélamo, N., Lacour, S., Tuthill, P., Ireland, M., Kraus, A., \& Chauvin, G. 2011, A\&A, 528, L7

Hughes, A. M., Wilner, D. J., Calvet, N., D'Alessio, P., Claussen, M. J., \& Hogerheijde, M. R. 2007, ApJ, 664, 536

Kim, K. H., Watson, D. M., Manoj, P., et al. 2013, ApJ, 769, 149
Kraus, A. L. \& Ireland, M. J. 2012, ApJ, 745, 5

Kraus, A. L., Ireland, M. J., Martinache, F., \& Hillenbrand, L. A. 2011, ApJ, 731, 8

Lin, D. N. C. \& Papaloizou, J. C. B. 1993, in Protostars and Planets III, ed. E. H. Levy \& J. I. Lunine, 749-835

Lissauer, J. J., Hubickyj, O., D'Angelo, G., \& Bodenheimer, P. 2009, Icarus, 199, 338

Lyra, W., Johansen, A., Klahr, H., \& Piskunov, N. 2009, A\&A, 493,1125

Mordasini, C., Alibert, Y., Klahr, H., \& Henning, T. 2012, A\&A, 547, A111

Movshovitz, N., Bodenheimer, P., Podolak, M., \& Lissauer, J. J. 2010, Icarus, 209, 616

Muzerolle, J., Allen, L. E., Megeath, S. T., Hernández, J., \& Gutermuth, R. A. 2010, ApJ, 708, 1107

Najita, J. R., Strom, S., \& Muzerolle, J. 2007, MNRAS, 378, 369

Owen, J. E. 2014, ApJ, 789, 59

Papaloizou, J. C. B. \& Nelson, R. P. 2005, A\&A, 433, 247

Pollack, J. B., Hubickyj, O., Bodenheimer, P., Lissauer, J. J., Podolak, M., \& Greenzweig, Y. 1996, Icarus, 124, 62

Quanz, S. P., Amara, A., Meyer, M. R., et al. 2014, arXiv: 1412.5173

Quanz, S. P., Amara, A., Meyer, M. R., Kenworthy, M. A., Kasper, M., \& Girard, J. H. 2013, ApJ, 766, L1

Reggiani, M., Quanz, S. P., Meyer, M. R., et al. 2014, ApJ, 792, L23

Rice, W. K. M., Armitage, P. J., Wood, K., \& Lodato, G. 2006, MNRAS, 373, 1619

Sallum, S., Eisner, J. A., Close, L. M., et al. 2015, arXiv:1501.01964

Skemer, A. J., Hinz, P., Esposito, S., et al. 2014, Proc. SPIE, 9148, 0

Spiegel, D. S. \& Burrows, A. 2012, ApJ, 745, 174 
Strom, K. M., Strom, S. E., Edwards, S., Cabrit, S., \& Skrutskie, M. F. 1989, AJ, 97, 1451

Varnière, P., Blackman, E. G., Frank, A., \& Quillen, A. C. 2006, ApJ, 640, 1110

Zhu, Z. 2015, ApJ, 799, 16
Zhu, Z., Nelson, R. P., Dong, R., Espaillat, C., \& Hartmann, L. 2012, ApJ, 755, 6

Zhu, Z., Nelson, R. P., Hartmann, L., Espaillat, C., \& Calvet, N. 2011, ApJ, 729, 47 